\title{
The proliferation marker Ki67, but not neuroendocrine expression, is an independent factor in the prediction of prognosis of primary prostate cancer patients
}

\author{
Mariarosa Pascale1, Cinzia Aversa², Renzo Barbazza², Barbara Marongiu¹, \\ Salvatore Siracusano ${ }^{2}$, Flavio Stoffel ${ }^{3}$, Sando Sulfaro ${ }^{4}$, Enrico Roggero ${ }^{1}$, Serena Bonin², \\ Giorgio Stanta² \\ ${ }^{1}$ Oncology Institute of Southern Switzerland (IOSI), Bellinzona, Switzerland \\ 2 Department of Medical Sciences, University of Trieste, Cattinara Hospital, Trieste, Italy \\ ${ }^{3}$ Department of Urology, Ospedale San Giovanni, Bellinzona, Switzerland \\ ${ }^{4}$ Department of Laboratory Medicine, S.C. Pathology, Santa Maria degli Angeli Hospital, Pordenone, Italy.
}

Radiol Oncol 2016; 50(3): 313-320

Received 8 February 2016

Accepted 28 April 2016

Correspondence to: Serena Bonin, M.D., Department of Medical Sciences, Cattinara University Hospital, Strada di Fiume 447, 34149 Trieste, Italy. Phone: +39 40 3996266; E-mail: sbonin@units.it

Disclosure: No potential conflicts of interest were disclosed.

Background. Neuroendocrine markers, which could indicate for aggressive variants of prostate cancer and Ki67 (a well-known marker in oncology for defining tumor proliferation), have already been associated with clinical outcome in prostate cancer. The aim of this study was to investigate the prognostic value of those markers in primary prostate cancer patients.

Patients and methods. NSE (neuron specific enolase), ChrA (chromogranin A), Syp (Synaptophysin) and Ki67 staining were performed by immunohistochemistry. Then, the prognostic impact of their expression on overall survival was investigated in 166 primary prostate cancer patients by univariate and multivariate analyses.

Results. NSE, ChrA, Syp and Ki67 were positive in 50, 45, 54 and 146 out of 166 patients, respectively. In Kaplan-Meier analysis only diffuse NSE staining (negative vs diffuse, $p=0.004$ ) and Ki67 ( $\leq 10 \%$ vs $>10 \%, p<0.0001$ ) were significantly associated with overall survival. Ki67 expression, but not NSE, resulted as an independent prognostic factor for overall survival in multivariate analysis.

Conclusions. A prognostic model incorporating Ki67 expression with clinical-pathological covariates could provide additional prognostic information. Ki67 may thus improve prediction of prostate cancer outcome based on standard clinical-pathological parameters improving prognosis and management of prostate cancer patients.

Key words: primary prostate cancer; prognosis; Ki67; NSE

\section{Introduction}

Conventional clinical parameters alone are inadequate for differentiating indolent and aggressive prostate cancer. Therefore, molecular biomarkers are needed to better define prognosis of prostate cancer patients.

Neuroendocrine markers could be used to detect particularly aggressive variants of prostate cancer.
Typical markers used to identify neuroendocrine differentiation (NED) in tumor tissue are neuron specific enolase (NSE), chromogranin A (CgA) and synaptophysin (Syp). ${ }^{1-3}$ Neuroendocrine differentiation, measured by one or more of those markers, has been associated with disease progression ${ }^{4}$ or poor survival in prostate cancer ${ }^{5}$, but up to now its prognostic value has not been clarified because of controversial results ${ }^{6 ; 7,8}$. However, Epstein et al. ${ }^{9}$ 
have recently suggested using neuroendocrine markers to better characterize and classify NED in prostate cancer. They also outlined Ki67 ranges in those tumors, which usually have a high proliferative index. ${ }^{4,9-13} \mathrm{Ki} 67$ is a well-known marker in oncology for defining tumor proliferation. Expression of Ki67 detection by immunohistochemistry ${ }^{14}$ is used as a prognostic marker for cell proliferation in many tumors, especially in breast carcinoma ${ }^{15}$ and cervical cancer. ${ }^{16}$ In prostate cancer it has also been associated with clinical outcome, irrespective of treatment. ${ }^{4,17-27}$

The aims of this study were to 1 ) investigate the immunohistochemical expression of neuroendocrine and Ki67 markers in primary prostate cancer patients in order to identify tumors characterized by biological aggressiveness and poor prognosis, 2) evaluate neuroendocrine expression with respect to Ki67 staining.

\section{Patients and methods}

\section{Patients}

Detailed histopathological and clinical data were retrospectively collected for 166 patients, who were diagnosed with primary prostate cancer in a single institution of the North-eastern Italy from January 1992 to December 1994, therefore associated to a long follow-up period. Inclusion criteria for this study were: a) diagnosis of prostate cancer and b) availability of formalin-fixed and paraffin-embedded tissues for immunohistochemical staining and molecular analyses. Only TURP ( $\mathrm{N}=122,73.9 \%)$ and prostatectomy $(\mathrm{N}=43,26.1 \%)$ specimens were used (missing information for one patient). Fine needle biopsies were excluded because of the low amount of tissue. Patients did not receive any treatment before diagnosis. The use of formalin-fixed and paraffin-embedded prostate cancer tissues and

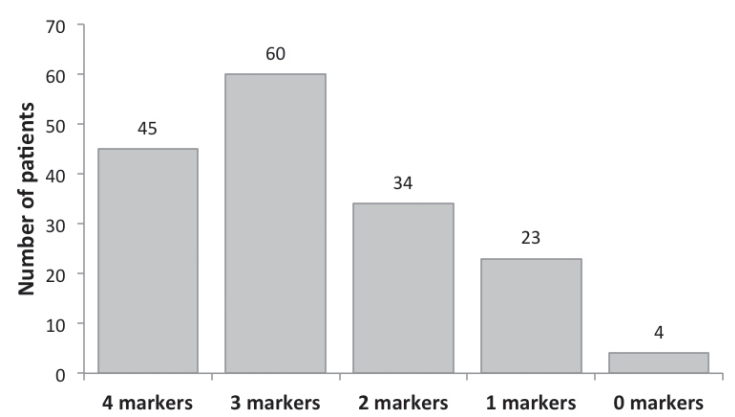

FIGURE 1. Determination of Ki67, NSE, CgA, SYP in primary prostate cancer patients. Number of concurrent biomarkers evaluated. The number of patients is reported above each column. their related clinical information were approved by the Ethical Committee of the University of Trieste (Report 23; 5.10.2009) before the beginning of the study.

\section{Tissue microarray and immunohistochemical staining}

Representative multiple areas of the primary tumours were selected by two pathologists (G.S. and R.B.) for TMA construction. Tissue cores were chosen at the border of the primary tumour. Tissue cylinders of $1.0 \mathrm{~mm}$ in diameter were taken from the selected regions of the donor's paraffin block and were placed into a recipient paraffin block using a tissue-arrayer (Galileo TMA CK3500; Integrated Systems Engineering, Milano, Italy), as previously described. ${ }^{28}$ Multiple cores were taken for cases as representative of heterogeneous histological areas. Neuroendocrine differentiation (NED) was evaluated using NSE, ChrA, Syp as neuroendocrine markers.

Immunostainings for Ki67 (clone MIB-1; DakoDenmark A/S, Glostrup, Denmark), 1:200 dilution; NSE (clone E27; NeoMarker; ThermoScientific, Waltham, MA, USA) 1:2500; ChrA (clone LK2H10; NeoMarker; ThermoScientific, Waltham, MA, USA), 1:500; Syp (clone SY 38; Thermo-Fisher; ThermoScientific, Waltham, MA, USA) 1:75 were performed in a Lab Vision Autostainer 480S (Thermo Scientific, Waltham, MA, USA) with the UltraVision LP Large Volume Detection System HRP Polymer (Lab Vision Corporation, Thermo Scientific) according to manufacturer's recommended protocol. For evaluation of the immunostaining, positively stained cells were counted across 3 high-power fields. Staining intensity was not taken into consideration. Due to technical issues related to the detachment of tissue cores it was not possible to analyze the four biomarkers in all samples (Figure 1). The percentage of the positively stained cells was reported for each specimen. Ki67 expression was dichotomized for assessing its prognostic value using a cut-off of $10 \% .^{7,29}$

\section{Statistical analysis}

The Kaplan-Meier method was used to generate overall survival (OS) curves, which was defined as the time between the date of diagnosis and the date of death or the last follow-up (FU) observation. Patients were censored if they were still alive or they were lost to FU. The log-rank test was used to evaluate differences between groups. Association 
TABLE 1. Neuroendocrine marker staining in our cohort of primary prostate cancer patients

\begin{tabular}{lccc}
\hline \multirow{2}{*}{ Staining } & \multicolumn{3}{c}{ Neuroendocrine markers } \\
\cline { 2 - 4 } & NSE & CgA & Syp \\
\hline Negative & $28(31.5)$ & $49(40.5)$ & $44(34.6)$ \\
Diffuse & $50(56.1)$ & $45(37.2)$ & $54(42.5)$ \\
Focal & $3(3.4)$ & $19(15.7)$ & $17(13.4)$ \\
Spotty & $8(9)$ & $8(6.6)$ & $12(9.5)$ \\
Total & 89 & 121 & 127 \\
missing & 77 & 45 & 39 \\
\hline
\end{tabular}

Data reported as $\mathrm{N}(\%)$

TABLE 2. Distribution of Ki67 positively stained cells in our cohort of primary prostate cancer patients

\begin{tabular}{lcc}
\hline \multirow{2}{*}{ \% Ki67-positive cells } & \multicolumn{2}{c}{ Number of samples } \\
\cline { 2 - 3 } & $\mathbf{N}$ & $\%$ \\
\hline 0 & 14 & 9.6 \\
$\leq 5$ & 70 & 47.9 \\
$>5$ and $\leq 10$ & 24 & 16.5 \\
$>10$ and $\leq 20$ & 18 & 12.3 \\
$>20$ and $\leq 30$ & 13 & 8.9 \\
$>30$ & 7 & 4.8 \\
Total & 146 & $100 \%$ \\
\hline
\end{tabular}

of OS with each prognostic factor was evaluate in univariate and multivariate analyses by using the Cox proportional hazards model. All variables associated with univariate value of $p \leq 0.05$ were included in the multivariate model using a stepwise method. The proportional hazards assumptions were checked before applying the Cox regression model. The goodness of fit was assessed using a likelihood-ratio test. The discrimination ability was quantified by calculating the concordance index that ranges from 0.5 (no discrimination) to 1 (perfect discrimination). Possible correlations of the expression of Ki67 and neuroendocrine markers with the other prognostic variables were assessed by $\chi 2$ test or Wilcoxon rank sum. All statistical tests were performed using STATA software (StataCorp. 2011. Stata Statistical Software: Release 12. College Station, TX: StataCorp LP) and $p$ values $\leq 0.05$ were considered as statistically significant.

\section{Results}

\section{Neuroendocrine marker staining}

NSE expression was assessable in 89 prostate cancer cases. Of those, 28 cases $(31.5 \%)$ were completely negative, whereas $61(68.5 \%)$ revealed a cytoplasmic positivity, referring to diffuse, focal and spotty staining (Table 1). Detachment of tissue

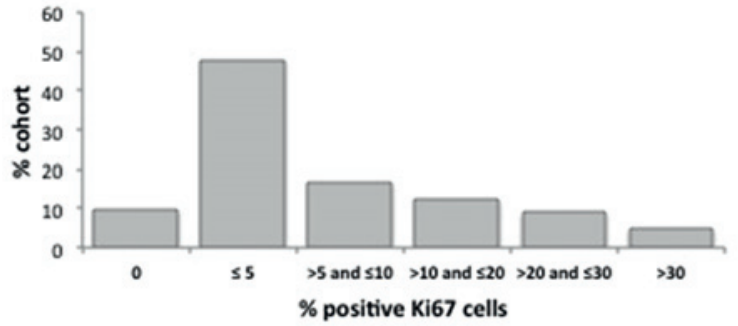

FIGURE 2. Distribution of Ki67 staining score. Positive Ki67 cells in TURP and prostatectomy specimens from a cohort of 166 primary prostate cancer patients.

TABLE 3. Association between Ki67 expression and clinicopathological variables ( $N=146)$

\begin{tabular}{|c|c|c|c|c|}
\hline \multirow{2}{*}{ Variable } & \multicolumn{4}{|c|}{ Ki67 expression } \\
\hline & $\leq 10 \%$ & $>10 \%$ & Tot & $P$-value \\
\hline $\begin{array}{l}\text { Nuclear grading } \\
1 \\
2 \\
3\end{array}$ & $\begin{array}{l}22 \\
72 \\
14\end{array}$ & $\begin{array}{c}1 \\
23 \\
14\end{array}$ & $\begin{array}{l}23 \\
95 \\
28\end{array}$ & $0.001^{*}$ \\
\hline $\begin{array}{l}\text { Gleason Score } \\
<7 \\
\geq 7 \\
\text { missing } 1\end{array}$ & $\begin{array}{l}56 \\
51\end{array}$ & $\begin{array}{c}8 \\
30\end{array}$ & $\begin{array}{l}64 \\
81\end{array}$ & $0.001^{*}$ \\
\hline $\begin{array}{l}\text { Age at diagnosis (median, years } \\
\quad \leq 71 \\
\quad>71\end{array}$ & $\begin{array}{l}53 \\
55\end{array}$ & $\begin{array}{l}19 \\
19\end{array}$ & $\begin{array}{l}72 \\
74\end{array}$ & 0.922 \\
\hline Age at diagnosis (continuous) & 108 & 38 & 146 & 0.973 \\
\hline $\begin{array}{l}\text { NSE expression } \\
\text { Negative } \\
\text { Positive }^{a} \\
\text { missing } 77\end{array}$ & $\begin{array}{l}21 \\
28\end{array}$ & $\begin{array}{c}4 \\
16\end{array}$ & $\begin{array}{l}25 \\
44\end{array}$ & 0.073 \\
\hline $\begin{array}{l}\text { CgA expression } \\
\text { Negative } \\
\text { Positive }^{a} \\
\text { missing } 40\end{array}$ & $\begin{array}{l}37 \\
24\end{array}$ & $\begin{array}{l}10 \\
12\end{array}$ & $\begin{array}{l}47 \\
36\end{array}$ & 0.217 \\
\hline $\begin{array}{l}\text { Syp expression } \\
\text { Negative } \\
\text { Positive } \\
\text { missing } 60\end{array}$ & $\begin{array}{l}31 \\
33\end{array}$ & $\begin{array}{c}8 \\
14\end{array}$ & $\begin{array}{l}39 \\
47\end{array}$ & 0.326 \\
\hline $\begin{array}{l}\text { Type of intervention } \\
\text { TURP } \\
\text { Prostatectomy } \\
\text { missing } 1\end{array}$ & $\begin{array}{l}78 \\
29\end{array}$ & $\begin{array}{c}33 \\
5\end{array}$ & $\begin{array}{c}111 \\
34\end{array}$ & 0.081 \\
\hline Total & 108 & 38 & 146 & \\
\hline
\end{tabular}

a Only diffuse expression was considered as positive staining; *Significant value

cores did not allow analyzing NSE in some cases. ChrA was negative in 49 out of 121 cores (40.5\%) and it was positive on the cytoplasmic level in 72 cores $(59.5 \%)$. Positive cases had weakly to highly diffused, or focal or spotty reactivity (Table 1). Syp was analyzed in 127 cases of which 44 (34.6\%) were negative and $83(65.4 \%)$ showed a cytoplasmic positivity, from spotty to focal to diffused (Table 1). Only diffused expression was considered as posi- 
TABLE 4. Univariate analysis of prognostic factors in patients with primary prostate cancer based on Cox proportional hazards regression model

\begin{tabular}{|c|c|c|}
\hline \multirow{2}{*}{ Variable } & \multicolumn{2}{|c|}{ UNIVARIATE } \\
\hline & $\mathrm{HR}(95 \% \mathrm{Cl})$ & $P$-value \\
\hline \multicolumn{3}{|l|}{ Nuclear grading } \\
\hline $\begin{array}{l}2 \text { vs } 1 \\
3 \text { vs } 1\end{array}$ & $\begin{array}{l}1.45(0.90-2.34) \\
3.49(1.94-6.27)\end{array}$ & $\begin{array}{r}0.125 \\
<0.001^{*}\end{array}$ \\
\hline \multicolumn{3}{|l|}{ Gleason score } \\
\hline$\geq 7$ vs $<7$ & $2.97(2.07-4.26)$ & $<0.001^{*}$ \\
\hline \multicolumn{3}{|l|}{ Ki67 expression } \\
\hline \multicolumn{3}{|l|}{ Age at diagnosis } \\
\hline (continuous) & $1.04(1.02-1.07)$ & $<0.001^{*}$ \\
\hline \multicolumn{3}{|l|}{ NSE expression } \\
\hline Positive ${ }^{a}$ vs negative & $1.99(1.19-3.34)$ & $0.009 *$ \\
\hline \multicolumn{3}{|l|}{ CgA expression } \\
\hline Positive a vs negative & $0.96(0.62-1.47)$ & 0.840 \\
\hline \multicolumn{3}{|l|}{ Syp expression } \\
\hline Positive ${ }^{a}$ vs negative & $1.18(0.77-1.81)$ & 0.447 \\
\hline \multicolumn{3}{|l|}{ Type of intervention } \\
\hline & $0.45(0.30-0.67)$ & $<0.001^{*}$ \\
\hline
\end{tabular}

$\mathrm{Cl}=$ confidence interval; $\mathrm{HR}=$ hazard ratio

a Only diffuse expression was considered as positive staining; *Significant value

TABLE 5. Multivariate analysis of prognostic factors in patients with primary prostate cancer based on Cox proportional hazards regression model stratified by type of intervention $(\mathrm{N}$ $=144$ )

\begin{tabular}{lcc}
\hline \multirow{2}{*}{ Variable } & \multicolumn{2}{c}{ MULTIVARIATE } \\
\cline { 2 - 3 } & $\begin{array}{c}\text { Adjusted } \\
\text { HR }(95 \% \mathrm{Cl})\end{array}$ & $\begin{array}{c}\text { Adjusted } \\
\text { P-value }\end{array}$ \\
\hline $\begin{array}{c}\text { Nuclear grading } \\
2 \text { vs } 1\end{array}$ & $1.27(0.75-2.13)$ & 0.373 \\
3 vs 1 & $1.93(1.01-3.68)$ & $0.045^{*}$ \\
Gleason score & & \\
$\geq 7$ vs $<7$ & $2.41(1.56-3.74)$ & $<0.001^{*}$ \\
Ki67 expression & & \\
$>10 \%$ vs $\leq 10 \%$ & $2.14(1.41-3.25)$ & $<0.001^{*}$ \\
$\begin{array}{l}\text { Age at diagnosis } \\
\text { (continuous) }\end{array}$ & $1.04(1.02-1.07)$ & $0.001^{*}$ \\
\hline
\end{tabular}

$\mathrm{Cl}=$ confidence interval; $\mathrm{HR}=$ hazard ratio; *Significant value

tive for each marker. Focal or spotty stainings were evaluated as negative.

No significant associations were found between neuroendocrine markers and clinicopathological variables. A slight association was revealed between NSE expression and Gleason score $(\mathrm{p}=0.04)$. Moreover, a positive relationship was found be- tween CgA and SYP expression ( $\mathrm{p}=0.01)$. The type of intervention was significantly associated with CgA $(p=0.009)$ and NSE $(p=0.001)$ expression.

\section{Ki67 staining}

Ki67 staining analysis was measured in 146 out of 166 prostatic cases. The median Ki67 staining score was $5 \%$ with an IQR of 9 whereas the mean value was $10.3 \%$ with a standard deviation of $14.2 \%$ (range of $0-90 \%$ ). The percentage of the positively stained cells was recorded for each sample (Table 2, Figures 2,3).

Ki67 was scored and stratified into two groups (low $\leq 10 \%$; high $>10 \%$ ) as already reported. ${ }^{23,24}$ No statistically significant difference was observed for Ki67 staining between TURP and prostatectomy specimens $(p=0.08)$. Ki67 expression was significantly associated with Gleason score and nuclear grading, but not with age at diagnosis or with neuroendocrine markers (Table 3 ).

\section{Univariate analysis}

In univariate analysis, diffuse expression of NSE, Ki67 expression $>10 \%$, Gleason score $\geq 7$ and nuclear grading $\geq 2$ predicted for shorter OS (Table 4).

Considering neuroendocrine markers, only NSE staining was significantly associated with OS. Patients with diffuse NSE expression had a reduced survival time (median OS 2 years; 95\% CI, 2-4) compared to patients with negative expression (median OS 7; 95\% CI, 5-10; $\mathrm{p}=0.004$ ), showing nearly double-fold increased risk of death (Table 4, Figure 4). Additionally, NED measured as positive at least at one of the three markers did not result significant.

Kaplan-Meier survival curves showed a significant difference $(\mathrm{p}<0.001)$ between low and high levels of Ki67 staining with median survival time of 6 years $(95 \% \mathrm{CI}, 5-9)$ and 2 years $(95 \% \mathrm{CI}$, $1-2)$ for patients with $\leq 10 \%$ and $>10 \%$ of positive cells, respectively (Figure 5A). Moreover, splitting group four categories according to Ki67 staining were obtained: negative, $1-10 \%, 11-20 \%$, > $20 \%$. Negative patients showed a median overall survival (10 years, 95\% CI: 4-9.) which was four years longer than in patients with Ki67 staining of $1-10 \%$ (6 years, $95 \%$ CI: $5-8 ; p<0.001$ ) (Figure $5 B$ ). An improved discrimination was also reached in the category $>10 \%$. Patients with Ki67 staining > $20 \%$ were associated with a 2.5 -fold increased risk of death, compared with patients showing Ki67 expression of $10-20 \%$ (the overall 2-year survival 
rate of $50 \%-95 \%$ CI: $25-70$ vs $20 \%$, $95 \%$ CI: $6-39$, p $<0.001)$. These data further support the potential role of Ki67 immunostaining in selecting patients according to proliferation rate, and thus to tumor aggressiveness. However, we decided to assess the prognostic value of Ki67 for overall survival of prostate cancer patients by using the previous binary variable because of harmonization with already published studies. ${ }^{7,29}$

Age at diagnosis and type of intervention significantly impacted on OS ( $\mathrm{p}<0.001)$. Thus, considering the relationship existing with other variables, they were included in multivariate analysis to take into account their possible confounding effect.

\section{Multivariate analysis}

Multivariate analysis was done by stratifying according to type of intervention, because of no proportional risks between TURP and prostatectomy groups (Test of proportional-hazards assumption, $\left.\chi^{2}=8.86, \mathrm{df}=1, \mathrm{p}=0.003\right)$. Ki67 expression, but not NSE, resulted as an independent prognostic factor for OS. In the final multivariable model the risk of death was higher for older patients $(p=0.001)$ with a nuclear grading of $3(\mathrm{p}=0.04)$, a Gleason score $\geq$ $7(p<0.001)$ and Ki67 expression $>10 \%(p<0.001)$ (Table 5). Unfortunately, our dataset did not include information on PSA before surgical intervention for all patients, because they were diagnosed many years ago before the routinely application of PSA screening.

Comparison of the multivariate model incorporating Ki67 expression with a base model including conventional variables only (nuclear grading, Gleason score, age at diagnosis stratified by type of intervention) showed an improved fit which suggested an enhanced prognostic ability over the models containing clinicopathological variables only $\left(\chi^{2}=11.33, \mathrm{df}=1, \mathrm{p}=0.0006\right)$. These data indicated that in a multivariate analysis Ki67 is a relevant and independent prognostic factor for OS of primary prostate cancer patients undergoing TURP or radical prostatectomy. The concordance index (0.72) revealed a good accuracy of the model in predicting OS.

\section{Discussion}

This study shows that Ki67 expression, but not NED, is an independent prognostic factor for OS in primary prostate cancer patients who underwent TURP or prostatectomy.
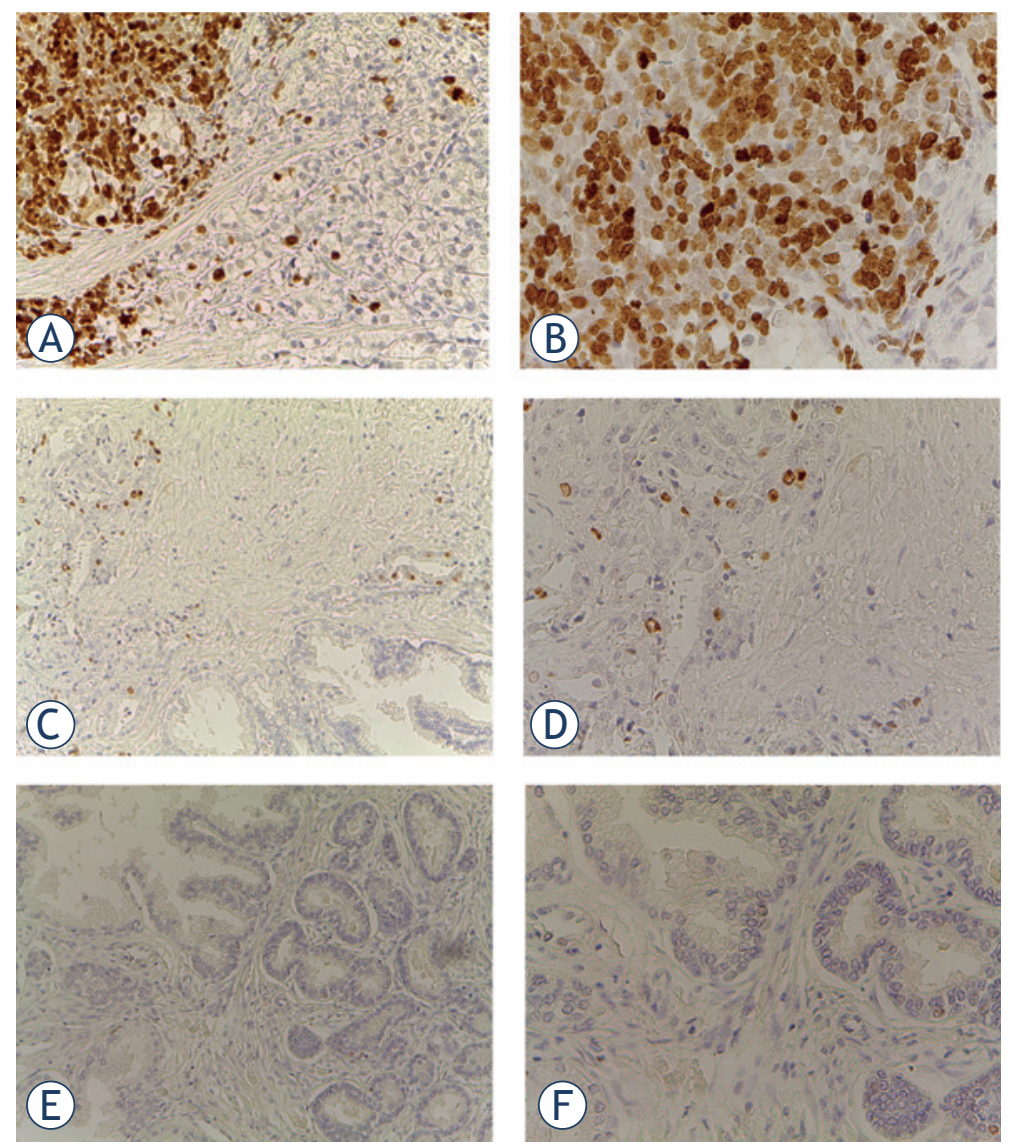

FIGURE 3. Representative immunohistochemical staining for Ki67. (A) prostate adenocarcinoma with $\mathrm{Ki} 67>10 \% 20 \times$ and $40 \times$ (B) magnification; (C) prostate adenocarcinoma with $\mathrm{Ki} 67 \leq 10 \% 20 \times$ and $40 \times$ (D) magnification; (E) prostate adenocarcinoma negative for Ki67 $20 \times$ and $40 \times(F)$ magnification.

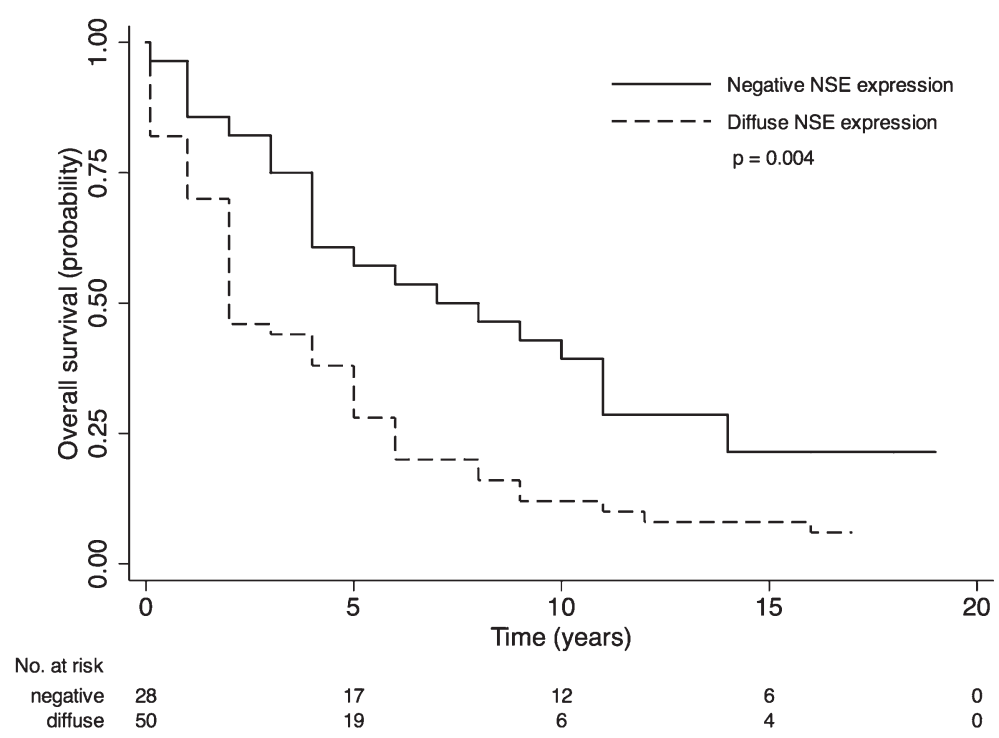

FIGURE 4. Survival curves by NSE staining in patients with primary prostate cancer. $\mathrm{p}$-value from log-rank test is reported. Numbers of at risk (still alive) patients are indicated below the $x$-axis. 
(A)

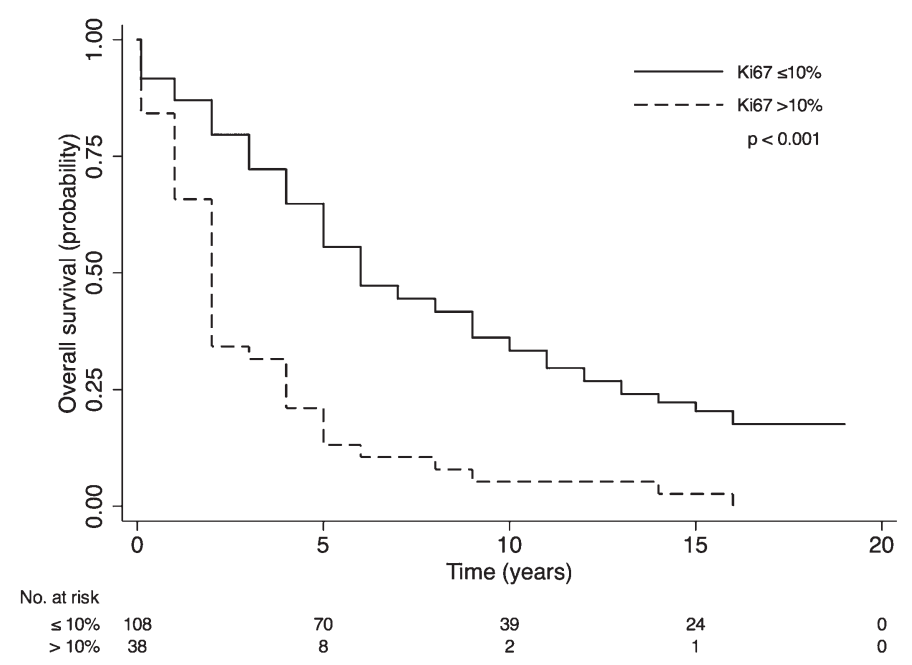

(B)

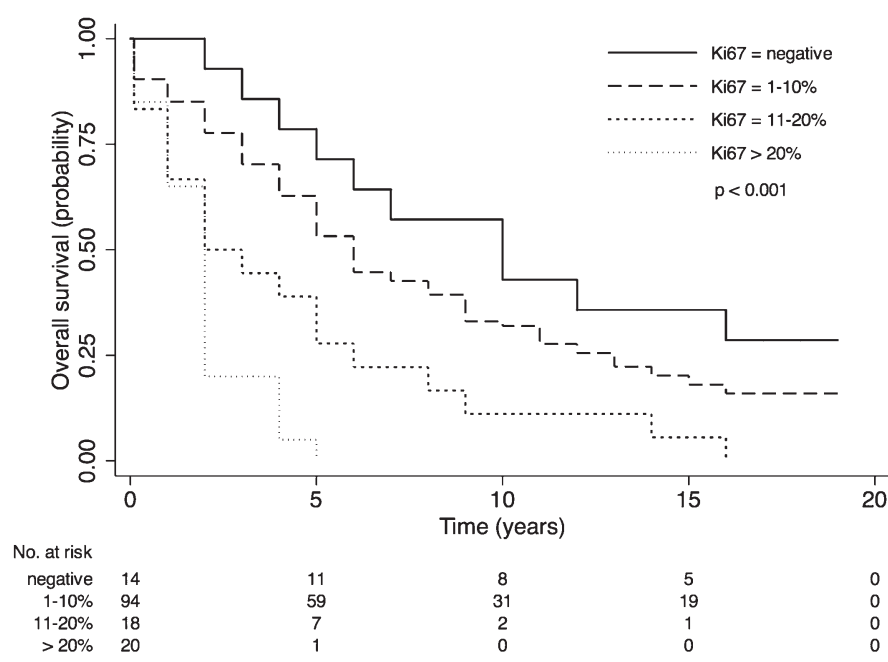

FIGURE 5. Survival curves by Ki67 expression in patients with primary prostate cancer. (A) Ki67 staining dichotomized in $\leq 10 \%$ and $>10 \%$; (B) Ki67 staining divided into negative, $1-10 \%, 11 \%-20 \%$, > 20\%. p-value from log-rank test is reported. Numbers of at risk (still alive) patients are indicated below the $x$-axis.

Our data demonstrate that NED, as measured by NSE, CgA and SYP immunohistochemistry, is present at the time of diagnosis in a large proportion of our cohort (over $50 \%$ ), but without influence on OS. NSE staining seems to influence OS, but it was not confirmed as an independent prognostic factor in the multivariate analysis. These results are consistent with current published literature where strong evidence of NSE as potential prognostic factor is lacking (reviewed in ${ }^{6,8}$ ), especially at early stages. ${ }^{30}$ NED in PCa increases with higher histological grades ${ }^{31}$ and disease progression, especially in response to androgen deprivation therapy. ${ }^{32-34}$ It seems that androgen deprivation therapy may promote the transformation of prostate adenocarcinoma into a neuroendocrine cancer, defined as t-NEPC (transformed neuroendocrine prostate cancer), as a mechanism of resistance. ${ }^{35} \mathrm{Ki} 67$ expression has already been proposed as a candidate marker for gastroenteropancreatic ${ }^{36}$ and lung neuroendocrine cancers. ${ }^{37}$ An increased proliferation using Ki67 expression has been shown in prostate tumors with high NED compared to tumors with low or without NED. ${ }^{4,10,11}$ In this study we did not find any association between NED and Ki67 expression, although a trend for a positive relationship between NSE staining and Ki67 expression ( $p$ $=0.08$ ) has been observed.

Limitations of this study are: small sample size, missing data for neuroendocrine IHC, statistically significant results obtained by using the less specific marker for NED. ${ }^{9}$ Another limitation is the analysis based on mixed sample population of prostatectomy and TURP which was partially solved using a stratified Cox model to investigate the risk difference in two groups. Of relevance, our cohort represents a long-term time series which allows investigating OS in cancers with a long life expectancy.

The prognostic significance of concurrent presence of NED features in prostate adenocarcinoma is currently very controversial. ${ }^{6}$ Although NED may have an adverse effect on prognosis of newly diagnosed prostate cancer, other mechanisms probably influence the prognosis by favoring the selection of the neuroendocrine pattern transformation under a specific stimulus, such as the pressure by androgen deprivation therapy. ${ }^{38}$ The mechanisms that are currently involved are not known.

Interestingly, we found that cell proliferation measured by Ki67 staining scored as a dichotomous variable with a $10 \%$ cut-off is an independent prognostic factor for OS in our cohort. Comparing prognostic models with and without Ki67 demonstrated that Ki67 expression could yield additional prognostic information to that provided by conventional clinicopathological parameters improving prognosis of prostate cancer patients. Furthermore, our data show that Ki67 expression correlates with Gleason score and nuclear grading, highlighting its association with prostate cancer aggressiveness, in agreement with others. , $23,25,39,40$ Several studies have shown that $\mathrm{Ki} 67$ is useful to predict prostate cancer prognosis either on TURP ${ }^{19,23}$ or needle biopsy $^{21,23,25,40}$ specimens. Furthermore, it has been proposed as a candidate prognostic marker both for overall and specific survival endpoints ${ }^{17-22}$ as well as disease progression. 4,17,18,20,22-27 Its prognostic rel- 
evance does not seem to be influenced by therapy, as it has been reported to predict prognosis in patients treated by prostatectomy, alone $e^{4,24,25,27}$ or with adjuvant therapy ${ }^{26}$, or radiotherapy, alone ${ }^{17,22,23}$ or with androgen deprivation therapy $17,18,20,22$, or conservatively managed. ${ }^{19,21}$

Consistent with our results, Ki67 emerges as a powerful marker of biological aggressiveness that could provide supplemental prognostic information, concerning the cellular proliferation rate, in addition to that provided by currently used markers, which are related to tumor pattern and extension only. Therefore, Ki67 may improve prediction of prostate cancer outcome based on standard clinical parameters, and may help stratify and select patients for more aggressive treatments. The utility of Ki67 was also demonstrated in selecting candidates with clinically insignificant cancer suitable for active surveillance among patients with PSA < $4 \mathrm{ng} / \mathrm{ml}$ at diagnosis. ${ }^{40}$ The validity of Ki67 expression as an indicator of prognosis for prostate cancer patients in different treatment cohorts, including both radical $l^{4,17,18,20,22-27}$ and conservative therapies $^{19,21}$, and in many investigative materials, such as biopsy ${ }^{21,23,25,40}$, TURP ${ }^{19,23}$ or prostatectomy ${ }^{4,24-27}$ specimens, further supports its implementation in clinical practice, as recently sustained also in a meta-analysis. ${ }^{41}$ However, larger prospective studies are needed to validate its use in routine pathology.

Despite unresolved issues on cut-offs, we suggest the analysis of Ki67 in routine diagnostic practice as an additional factor for improving prognosis and management of prostate cancer patients.

\section{Acknowledgments}

This work was partially supported by grants from ABREOC 2011.

\section{References}

1. Schmechel D, Marangos PJ, Brightman M. Neurone-specific enolase is a molecular marker for peripheral and central neuroendocrine cells. Nature 1978; 276: 834-6.

2. Kimura N, Funakoshi A, Aunis D, Tateishi K, Miura W, Nagura H. Immunohistochemical localization of chromostatin and pancreastatin, chromogranin A-derived bioactive peptides, in Normal and Neoplastic Neuroendocrine Tissues. Endocr Pathol 1995; 6: 35-43.

3. Wiedenmann B, Franke WW, Kuhn C, Moll R, Gould VE. Synaptophysin: a marker protein for neuroendocrine cells and neoplasms. Proc Natl Acad Sci USA 1986; 83: 3500-4.

4. May M, Siegsmund M, Hammermann F, Loy V, Gunia S. Prognostic significance of proliferation activity and neuroendocrine differentiation to predict treatment failure after radical prostatectomy. Scand J Urol Nephrol 2007; 41: $375-81$.
5. Krauss DJ, Amin M, Stone B, Ye H, Hayek S, Cotant M, et al. Chromogranin A staining as a prognostic variable in newly diagnosed Gleason score. Prostate 2014; 74: 520-7.

6. Berruti A, Vignani F, Russo L, Bertaglia V, Tullio M, Tucci M, et al. Prognostic role of neuroendocrine differentiation in prostate cancer, putting together the pieces of the puzzle. Open Access J Urol 2010; 2: 109-24.

7. Cindolo L, Cantile M, Franco R, Chiodini P, Schiavo G, Forte I, et al. Parallel determination of NeuroD1, chromogranin-A, KI67 and androgen receptor expression in surgically treated prostate cancers. Int Braz J Urol 2011; 37: 57-66.

8. Jeetle SS, Fisher G, Yang ZH, Stankiewicz E, Moller H, Cooper CS, et al. Neuroendocrine differentiation does not have independent prognostic value in conservatively treated prostate cancer. Virchows Arch 2012; 461: $103-7$.

9. Epstein JI, Amin MB, Beltran H, Lotan TL, Mosquera J-M, Reuter VE, et al Proposed morphologic classification of prostate cancer with neuroendocrine differentiation. Am J Surg Pathol 2014; 38: 756-67.

10. Helpap B, Kollermann J. Undifferentiated carcinoma of the prostate with small cell features: immunohistochemical subtyping and reflections on histogenesis. Virchows Arch 1999; 434: 385-91.

11. Grobholz R, Griebe M, Sauer CG, Michel MS, Trojan L, Bleyl U. Influence of neuroendocrine tumor cells on proliferation in prostatic carcinoma. Hum Pathol 2005; 36: 562-70

12. Gunia S, Albrecht K, Koch S, Herrmann T, Ecke T, Loy V, et al. Ki67 staining index and neuroendocrine differentiation aggravate adverse prognostic parameters in prostate cancer and are characterized by negligible interobserver variability. World J Urol 2008; 26: 243-50.

13. Beltran H, Tomlins S, Aparicio A, Arora V, Rickman D, Ayala G, et al. Aggressive variants of castration-resistant prostate cancer. Clin Cancer Res 2014; 20: 2846-50.

14. Gerdes J, Schwab U, Lemke H, Stein H. Production of a mouse monoclonal antibody reactive with a human nuclear antigen associated with cell proliferation. Int J Cancer 1983; 31: 13-20.

15. Dowsett M, Nielsen TO, A'Hern R, Bartlett J, Coombes RC, Cuzick J, et al. Assessment of Ki67 in Breast Cancer: Recommendations from the International Ki67 in Breast Cancer Working Group. J Natl Cancer Inst 2011; 103: $1656-64$.

16. Ikenberg H, Bergeron C, Schmidt D, Griesser H, Alameda F, Angeloni C, et al. Screening for cervical cancer precursors with p16/Ki-67 dual-stained cytology: results of the PALMS study. J Nat/ Cancer Inst 2013; 105: 1550-7.

17. Li R, Heydon K, Hammond ME, Grignon DJ, Roach M 3rd, Wolkov HB, et al. Ki-67 staining index predicts distant metastasis and survival in locally advanced prostate cancer treated with radiotherapy: an analysis of patients in radiation therapy oncology group protocol 86-10. Clin Cancer Res 2004; 10: $4118-24$

18. Pollack A, DeSilvio M, Khor LY, Li R, Al-Saleem TI, Hammond ME, et al. Ki-67 staining is a strong predictor of distant metastasis and mortality for men with prostate cancer treated with radiotherapy plus androgen deprivation: Radiation Therapy Oncology Group Trial 92-02. J Clin Oncol 2004; 22: 2133-40.

19. Berney DM, Gopalan A, Kudahetti S, Fisher G, Ambroisine L, Foster CS, et al. Ki-67 and outcome in clinically localised prostate cancer: analysis of conservatively treated prostate cancer patients from the Trans-Atlantic Prostate Group study. Br J Cancer 2009; 100: 888-93.

20. Khor LY, Bae K, Paulus R, Al-Saleem T, Hammond ME, Grignon DJ, et al. MDM2 and Ki-67 predict for distant metastasis and mortality in men treated with radiotherapy and androgen deprivation for prostate cancer: RTOG 9202. J Clin Oncol 2009; 27: 3177-84.

21. Fisher G, Yang ZH, Kudahetti S, Møller H, Scardino P, Cuzick J, et al Prognostic value of $\mathrm{Ki}-67$ for prostate cancer death in a conservatively managed cohort. Br J Cancer 2013; 108: 271-7.

22. Verhoven $B$, Yan $Y$, Ritter $M$, Khor $L Y$, Hammond $E$, Jones $C$, et al. Ki-67 is an independent predictor of metastasis and cause-specific mortality for prostate cancer patients treated on Radiation Therapy Oncology Group (RTOG) 94-08. Int J Radiat Oncol Biol Phys 2013; 86: 317-23.

23. Cowen D, Troncoso P, Khoo VS, Zagars GK, von Eschenbach AC, Meistrich $\mathrm{ML}$, et al. Ki-67 staining is an independent correlate of biochemical failure in prostate cancer treated with radiotherapy. Clin Cancer Res 2002; 8: 1148-54. 
24. Laitinen S, Martikainen PM, Tolonen T, Isola J, Tammela TL, Visakorpi T. EZH2, Ki-67 and MCM7 are prognostic markers in prostatectomy treated patients. Int J Cancer 2008; 122: 595-602.

25. Zellweger T, Günther S, Zlobec I, Savic S, Sauter G, Moch H, et al. Tumour growth fraction measured by immunohistochemical staining of Ki67 is an independent prognostic factor in preoperative prostate biopsies with small-volume or low-grade prostate cancer. Int J Cancer 2009; 124: 2116-23.

26. Antonarakis ES, Keizman D, Zhang Z, Gurel B, Lotan TL, Hicks JL, et al. An immunohistochemical signature comprising PTEN, MYC, and Ki67 predicts progression in prostate cancer patients receiving adjuvant docetaxel after prostatectomy. Cancer 2012; 118: 6063-71.

27. Rubio J, Ramos D, López-Guerrero JA, Iborra I, Collado A, Solsona E, et al Immunohistochemical expression of Ki-67 antigen, cox-2 and $\mathrm{Bax} / \mathrm{Bcl}-2$ in prostate cancer; prognostic value in biopsies and radical prostatectomy specimens. Eur Urol 2005; 48: 745-51.

28. Pascale M, Pracella D, Barbazza R, Marongiu B, Roggero E, Bonin S, et al. Is human papillomavirus associated with prostate cancer survival? Dis Markers 2013; 35: 607-13.

29. Zellweger T, Ninck C, Mirlacher M, Annefeld M, Glass AG, Gasser TC, et al. Tissue microarray analysis reveals prognostic significance of syndecan-1 expression in prostate cancer. Prostate 2003; 55: 20-9.

30. Surcel $\mathrm{Cl}$, van Oort IM, Sooriakumaran P, Briganti A, De Visschere PJL, Futterer JJ, et al. Prognostic effect of neuroendocrine differentiation in prostate cancer: A critical review. Urol Oncol 201; 33: 265.e1-7.

31. Hirano $D$, Jike $T$, Okada $Y$, Minei $S$, Sugimoto $S$, Yamaguchi $K$, et al. Immunohistochemical and ultrastructural features of neuroendocrine differentiated carcinomas of the prostate: an immunoelectron microscopic study. Ultrastruct Pathol 2005; 29: 367-75.

32. Hirano D, Okada Y, Minei S, Takimoto $Y$, Nemoto N. Neuroendocrine differentiation in hormone refractory prostate cancer following androgen deprivation therapy. Eur Urol 2004; 45: 586-92. discussion 592.

33. Beltran H, Tagawa ST, Park K, MacDonald T, Milowsky MI, Mosquera JM, et al. Challenges in recognizing treatment-related neuroendocrine prostate cancer. J Clin Oncol 2012; 30: e386-9.

34. Komiya A, Yasuda K, Watanabe A, Fujiuchi Y, Tsuzuki T, Fuse H. The prognostic significance of loss of the androgen receptor and neuroendocrine differentiation in prostate biopsy specimens among castration-resistant prostate cancer patients. Mol Clin Oncol 2013; 1: 257-62.

35. Aggarwal R, Zhang T, Small EJ, Armstrong AJ. Neuroendocrine prostate cancer: subtypes, biology, and clinical outcomes. J Natl Compr Canc Netw 2014; 12: 719-26.

36. Ezziddin S, Attassi M, Yong-Hing CJ, Ahmadzadehfar H, Willinek W, Grünwald $\mathrm{F}$, et al. Predictors of long-term outcome in patients with well-differentiated gastroenteropancreatic neuroendocrine tumors after peptide receptor radionuclide therapy with 177Lu-Octreotate. J Nucl Med 2014; 55: 183-90.

37. Pelosi G, Rindi G, Travis WD, Papotti M. Ki-67 antigen in lung neuroendocrine tumors: unraveling a role in clinical practice. J Thorac Oncol 2014; 9: $273-84$.

38. Mosquera JM, Beltran H, Park K, MacDonald TY, Robinson BD, Tagawa ST, et al. Concurrent AURKA and MYCN gene amplifications are harbingers of lethal treatment-related neuroendocrine prostate cancer. Neoplasia 2013, 15: $1-10$.

39. Khoo VS, Pollack A, Cowen D, Joon DL, Patel N, Terry NH, et al. Relationship of Ki-67 labeling index to DNA-ploidy, S-phase fraction, and outcome in prostate cancer treated with radiotherapy. Prostate 1999; 41: 166-72.

40. Nagao K, Yamamoto Y, Hara T, Komatsu H, Inoue R, Matsuda K, et al. Ki67 and BUBR1 may discriminate clinically insignificant prostate cancer in the PSA range < 4 ng/ml. Jpn J Clin Oncol 2011; 41: 555-64.

41. Zhao L, Yu N, Guo T, Hou Y, Zeng Z, Yang X, et al. Tissue biomarkers for prognosis of prostate cancer: a systematic review and meta-analysis. Cancer Epidemiol Biomarkers Prev 2014; 23: 1047-54. 